\title{
Aspectos clínicos e laboratoriais em ovinos submetidos a dietas com níveis elevados de enxofre com objetivo de indução de polioencefalomalácia ${ }^{1}$
}

\author{
Diego J.Z. Delfiol ${ }^{2}$, Didier Q. Cagnini ${ }^{2}$, Paulo H.J. Cunha ${ }^{3}$, Nadia Crosignani ${ }^{4}$, Angélica \\ T.B. Wouters ${ }^{5}$, Flademir Wouters ${ }^{6}$, David Driemeier ${ }^{5}$ e Alexandre S. Borges ${ }^{2 *}$
}

\begin{abstract}
Delfiol D.J.Z., Cagnini D.Q., Cunha P.H.J., Crosignani N., Wouters A.T.B., Wouters F., Driemeier D. \& Borges A.S. 2013. [Clinical and laboratory aspects of sheep supplemented with high levels of sulfur in diet to induce polioencephalomalacia.] Aspectos clínicos e laboratoriais em ovinos submetidos a dietas com níveis elevados de enxofre com objetivo de indução de polioencefalomalácia. Pesquisa Veterinária Brasileira 33(4):435-442. Departamento de Clínica Veterinária, Faculdade de Medicina Veterinária e Zootecnia, Universidade Estadual Paulista, Botucatu, SP 18618970, Brazil. E-mail: asborges@fmvz.unesp.br

Polioencephalomalacia (PEM) is a neuropathologic condition of ruminants that can be induced by a variety of factors including excessive sulfur intake. This study aimed to investigate the relationship between diets rich in sulfur, high levels of ruminal hydrogen sulfide and the occurrence of polioencephalomalacia in sheep. Eighteen sheep were divided into three groups (G1, G2, and G3) and supplemented with $0.2 \%, 0.9 \%$ and $1.2 \%$ sulfur in the diet respectively. Clinical evaluation (i.e. heart rate, respiratory rate, rectal temperature and rumen motility) and laboratory exams (i.e. ruminal hydrogen sulfide concentration, venous gas analysis, ruminal $\mathrm{pH}$, serum and liver copper concentration, computed axial tomography, necropsy, and histopathological examination) were performed. Rectal temperature, venous gas and ruminal $\mathrm{pH}$ were within normal limits. Tachycardia and tachypnea were observed in sheep of the three groups. Rumen motility was decreased in animals of group G2 and G3 when compared with G1. The higher the sulfur intake, the lower was the serum and liver levels of copper. Increased ruminal hydrogen sulfide concentration was detected in G2 and G3 sheep. None of the animals had clinical signs of PEM. Computed axial tomography, macroscopic and histopathological examination of the central nervous system showed no evidence of PEM. It is suggested that other factors are associated with excessive sulfur consumption for a PEM outbreak to occur in sheep.
\end{abstract}

INDEX TERMS: Cerebrocortical necrosis, polioencephalomalacia, sulfur, ruminal hydrogen sulfide, sheep.

\footnotetext{
${ }^{1}$ Recebido em 22 de janeiro de 2013.

Aceito para publicação em 18 de fevereiro de 2013.

${ }^{2}$ Departamento de Clínica Veterinária, Faculdade de Medicina Veterinária e Zootecnia, Universidade Estadual Paulista (Unesp), Botucatu, SP 18618-970, Brasil. *Autor para correspondência: asborges@fmvz.unesp.br

${ }^{3}$ Departamento de Medicina Veterinária, Escola de Veterinária e Zootecnia, Universidade Federal de Goiás (UFG), Campus Samambaia (Campus II), Cx. Postal 131, Goiânia, G0 74001-970, Brasil.

${ }^{4}$ Departamento de Cirurgia e Anestesiologia Veterinária, Faculdade de Medicina Veterinária e Zootecnia, Unesp, Botucatu, SP.

${ }^{5}$ Departamento de Patologia Clínica Veterinária, Faculdade de Veterinária, Universidade Federal do Rio Grande do Sul (UFRGS), Av. Bento Gonçalves 9090, Porto Alegre, RS 95320-000, Brasil.

${ }^{6}$ Departamento de Patologia Veterinária, Universidade Federal de Lavras (UFLA), Cx. Postal 3037, Lavras, MG 37200-000, Brasil
}

RESUMO.- A polioencefalomalacia (PEM) é uma doença neurológica que acomete ruminantes e pode ser desencadeada por diversos fatores, dentre eles o consumo excessivo de enxofre. Este trabalho teve como objetivo verificar a relação entre dietas ricas em enxofre, altos níveis de gás sulfídrico ruminal e a ocorrência de polioencefalomalácia em ovinos. Foram utilizados 18 ovinos, divididos em três grupos (G1, G2 e G3) que receberam diferentes níveis de enxofre na dieta; $0,2 \%, 0,9 \%$ e 1,2\%, respectivamente. Exames físicos (frequência cardíaca, frequência respiratória, temperatura retal e motricidade ruminal) e complementares (concentração de sulfeto de hidrogênio ruminal, hemogasometria venosa, $\mathrm{pH}$ do fluído ruminal, concentração de cobre sérico e hepático, tomografia computadorizada, ne- 
cropsia e histopatologia) foram realizados. A temperatura retal, a hemogasometria venosa e o $\mathrm{pH}$ do fluido ruminal permaneceram dentro dos valores de referência para a espécie. A motricidade ruminal estava diminuída nos grupos G2 e G3 em comparação com o G1 (controle). Quanto maior a ingestão de enxofre, menores foram os níveis de cobre sérico e hepático. Valores elevados de sulfeto de hidrogênio ruminal foram detectados nos grupos G2 e G3. Nenhum animal apresentou sinais clínicos de PEM. Nos exames de tomografia computadorizada, necropsia e exame histopatológico do sistema nervoso central (SNC), não foram observadas alterações compatíveis com PEM. É provável que algum outro fator esteja associado ao excesso de enxofre na dieta para o desenvolvimento de PEM em ovinos.

TERMOS DE INDEXAÇÃO: Necrose cerebrocortical, polioencefalomalácia, enxofre, sulfeto de hidrogênio ruminal, ovinos.

\section{INTRODUÇÃO}

0 enxofre é um macroelemento que tem grande importância metabólica na nutrição de ruminantes, pois é componente dos aminoácidos metionina e cisteína, de proteínas e de uma série de outros compostos orgânicos (Burgess 2008). Os níveis de enxofre recomendados na dieta de ovinos estão entre 0,14 e 0,26\% da matéria seca (NRC 2005). Em contrapartida, a ingestão excessiva desse mineral pode diminuir o ganho de peso dos animais, causar problemas respiratórios, entéricos e neurológicos (Alves de Oliveira et al. 1996, Bulgin et al. 1996, Kung Jr 2008).

A PEM é uma doença neurológica que acomete ruminantes (Jensen et al. 1956), caracterizada por necrose cerebrocortical e que pode evoluir para malácia (Gould 1998). Em ovinos, a doença pode ser causada por deficiência ou distúrbios no metabolismo da tiamina (Jensen et al. 1956, Sant'Ana et al. 2009a), pela intoxicação por sal associada à privação de água (Scarratt et al. 1985), pelo consumo excessivo de enxofre na dieta (Gooneratne et al. 1989) e pela inoculação experimental do Herpesvírus bovino 5 (Silva et al. 1998).

A literatura internacional aponta o consumo excessivo de enxofre como importante causa de PEM em bovinos e ovinos (Gould 2000). A doença em ovinos tem ocorrido em forma de surtos (Jeffrey et al. 1994, Bulgin et al. 1996, Low et al. 1996) ou tem sido induzida experimentalmente (Gooneratne et al. 1989, Rousseaux et al. 1991, McAllister et al. 1992, Alves de Oliveira et al 1996, Krasicka et al. 1999, Salem et al., 2010). No Brasil, a PEM ocasionada pelo consumo excessivo de enxofre foi descrita em bovinos na forma de surtos (Traverso et al. 2001, Cunha et al. 2010) e em indução experimental (Cunha et al. 2011). Existe apenas um surto de PEM em ovinos relatado no Brasil, em que os autores estimaram que os animais estivessem consumindo quantidades excessivas de enxofre na dieta (Lima et al. 2005).

A técnica de determinação da concentração do sulfeto de hidrogênio ruminal $\left(\mathrm{H}_{2} \mathrm{~S}\right)$ com utilização de tubos colorimétricos tem sido empregada com sucesso em bovinos para identificar rebanhos que estejam ingerindo alto teor de enxofre na dieta (Gould et al. 1997, Cunha et al. 2010,
2011). Em ovinos, a técnica foi utilizada somente por Neville et al. (2010), que identificaram altos níveis de $\mathrm{H}_{2} \mathrm{~S}$ ruminal em animais em dieta com alto teor de enxofre, apesar dos ovinos não terem desenvolvido PEM.

Confirmar a PEM induzida por consumo excessivo de enxofre em ruminantes é uma tarefa desafiadora e tem estimulado o desenvolvimento de novos modelos experimentais para melhor compreensão da doença (Cunha et al. 2011, Drewnoski et al 2012). Deve se ressaltar que não se sabe por que ovinos e bovinos alimentados com dietas ricas em enxofre e com valores elevados de sulfeto de hidrogênio ruminal (Neville et al. 2010, Drewnoski et al 2012,), podem não desenvolver sinais neurológicos e lesões no SNC.

Este trabalho tem como objetivo verificar a relação entre dietas ricas em enxofre, altos níveis de gás sulfídrico ruminal e a ocorrência de polioencefalomalácia em ovinos.

\section{MATERIAL E MÉTODOS}

Foram utilizados 18 cordeiros machos, mestiços da raça Santa Inês, com aproximadamente quatro meses de idade e peso médio de $27 \pm 3 \mathrm{~kg}$. Os animais foram distribuídos aleatoriamente em três grupos experimentais com seis animais e mantidos em baias com $12 \mathrm{~m}^{2}$, durante 111 dias de experimento, com três animais de um mesmo grupo em cada baia. Uma semana antes do início do estudo os animais foram pesados e everminados com levamisol na dose de 7,5mg/kg de peso.

0 protocolo de indução de PEM foi adaptado de Cunha et al. (2011). 0 experimento foi dividido em três fases: na fase 1 (adaptação) os animais receberam feno de Coast-cross (Cynodon dactylon) por 14 dias. Na fase 2 (transição) que durou sete dias, o feno foi substituído gradativamente pela ração. Na fase 3 os ovinos consumiram uma dieta constituída por $95 \%$ da ração experimental e $5 \%$ de feno de Coast-cross por 90 dias. As sobras da ração eram pesadas todos os dias para mensurar a quantidade ingerida pelos animais.

A ração experimental era composta por 30,8\% de casca de aveia, $18,5 \%$ de farelo de soja, $17 \%$ de amido de milho, $17 \%$ de dextrose, $4 \%$ de óleo de milho, $3 \%$ de uréia, $2 \%$ de bicarbonato de sódio, $2,5 \%$ de fosfato bicálcico, $0,5 \%$ de cloreto de potássio, $0,2 \%$ de óxido de magnésio e 4,5\% de água, com adição das vitaminas, A 2000, D 100 e E 20 (UI/Kg de ração), conforme descrito por Sager et al. (1990).

Cada grupo recebeu um nível diferente de enxofre na ração experimental. No grupo 1 (G1; controle) a ração continha $0,2 \%$ de enxofre na matéria seca (MS). Nos grupos 2 (G2) e 3 (G3) adicionou-se sulfato de sódio à ração de modo que as concentrações finais de enxofre ficassem em $0,9 \%$ na MS para o grupo 2 e 1,2\% na MS para o grupo 3.

Amostras de água ${ }^{7}$, feno $^{8}$ e ração ${ }^{9}$ foram colhidas antes do início do experimento para determinação do teor de enxofre. As amostras do feno e da ração foram submetidas às análises bromatológica ${ }^{10}$ e mineral ${ }^{9}$. Além disso, realizou-se dosagem de tiamina $^{11}$ na ração experimental por cromatografia líquida de alta eficiência (HPLC).

\footnotetext{
${ }^{7}$ Laboratório de Fertilizantes e Corretivos, Faculdade de Ciências Agronômicas da Unesp/Botucatu.

${ }^{8}$ Laboratórios de Nutrição Mineral de Plantas da FMVZ/Unesp/Botucatu.

${ }^{9}$ Instituto Campineiro de Análise de Solo e Adubo Ltda.

${ }^{10}$ Laboratório de Bromatologia da FMVZ/Unesp/Botucatu.

${ }^{11}$ CCQA-ITAL, Instituto de Tecnologia de Alimentos.
} 
Durante o experimento os animais foram avaliados clinicamente quatro vezes ao dia para possíveis alterações neurológicas (nível de consciência, alteração de comportamento, postura e alterações no padrão de locomoção). Caso alguma alteração fosse observada o ovino seria submetido ao exame neurológico conforme descrito por De Lahunta (2009).

Os animais foram examinados e realizou-se colheita de amostras no primeiro e no décimo quarto dias da fase 1 (M1 e M2), no sétimo dia da fase 2 (M3) e de dez em dez dias na fase 3 (M4 a M12), totalizando 12 momentos. Em cada momento colheu-se sangue total para obtenção de soro, sangue venoso para realização de hemogasometria, líquido ruminal para aferição do pH e também foi dosada a concentração de $\mathrm{H}_{2} \mathrm{~S}$ ruminal.

Nos momentos M1, M3, M5, M7, M9, M11 e M12 os animais foram pesados. E nos momentos M1 e M3 realizou-se exame coproparasitológico pela técnica de McMaster.

Para a obtenção de soro, amostras sanguíneas foram colhidas em tubos sem anticoagulante. Após a retração do coágulo, as amostras foram imediatamente centrifugadas e os soros armazenados em tubos plásticos e congelados $\left(-20^{\circ} \mathrm{C}\right)$ até a realização da dosagem de cobre.

Para a determinação da concentração de $\mathrm{H}_{2} \mathrm{~S}$ ruminal foi realizada ruminocentese utilizando a técnica descrita por Cunha et al. (2011). Foi realizada tricotomia na fossa paralombar esquerda e antissepsia com iodopovidona (PVPI). A punção ruminal foi realizada no ponto médio da porção mais dorsal da fossa paralombar esquerda, com a agulha do cateter intravenoso 14G. Em seguida, foi encaixado um equipo intravenoso (15 $\mathrm{cm}$ de comprimento) ao conector da agulha. A ponta cortada do equipo adaptado foi acoplada ao tubo colorimétrico de dosagem de gás sulfídrico, previamente inserido na bomba manual de vácuo ${ }^{12}$. As mensurações foram realizadas de acordo com o manual do fabricante da bomba de vácuo, utilizando-se tubos de dosagem entre 25 a $1.000 \mathrm{ppm}^{13}$ e de 1.000 a $40.000^{14} \mathrm{ppm}$ padronizando $100 \mathrm{ml}$ de gás para cada amostra de $\mathrm{H}_{2} \mathrm{~S}$, com tempo de amostragem de 1,5 minutos.

As amostras sanguíneas para realização do exame hemogasométrico foram colhidas por venopunção da jugular externa, utilizando uma seringa de $1 \mathrm{~mL}$ heparinizada acoplada a uma agulha $30 x 8$. 0 exame foi realizado com um analisador portátil ${ }^{15}$ no qual era inserido o cartucho de leitura ${ }^{16}$ responsável por fornecer os resultados de pH sanguíneo, pressão de $\mathrm{CO}_{2}$ sanguíneo $\left(\mathrm{PCO}_{2}\right)$, pressão de $\mathrm{O}_{2}$ sanguíneo $\left(\mathrm{PO}_{2}\right)$, excesso de bases (BE), bicarbonato $\left(\mathrm{HCO}_{3}^{-}\right)$, total de $\mathrm{CO}_{2}$ sanguíneo $\left(\mathrm{tCO}_{2}\right)$, saturação do oxigênio $\left(\mathrm{sO}_{2}\right)$, sódio $\left(\mathrm{Na}^{+}\right)$, potássio $\left(\mathrm{K}^{+}\right)$, cálcio ionizado $\left(\mathrm{iCa}^{2+}\right)$, hematócrito (Hct) e hemoglobina (Hb).

As amostras de líquido ruminal para aferição do $\mathrm{pH}$ foram colhidas quatro horas após o fornecimento da ração, utilizando-se uma bomba de sucção a vácuo, acoplada a um recipiente de vidro e a uma sonda oroesofágica siliconizada de 12mm (Dirksen et al. 1993). Para evitar contaminação com saliva os primeiros $40 \mathrm{~mL}$ eram desprezados e colhidos mais $40 \mathrm{ml}$ para análise imediata ${ }^{17}$.

No nonagésimo dia da fase 3 (M12), quatro animais de cada grupo foram induzidos à anestesia geral com cetamina $(5 \mathrm{mg} / \mathrm{kg}$ I.V). Após a intubação, a manutenção do plano anestésico foi realizada com isoflurano em circuito com reinalação, usando $100 \%$ de oxigênio (Riebold 2007). Anestesiados, os ovinos foram submetidos ao exame de tomografia computadorizada contrastada do encéfalo ${ }^{18}$.

Após o exame de tomografia computadorizada todos os animais foram eutanasiados com xilazina $(0,10 \mathrm{mg} / \mathrm{kg}$ I.V.), tiopental sódico (8mg/kg I.V.) e por último, cloreto de potássio. Imediatamente após a eutanásia, realizou-se necropsia, com registro das alterações anatomopatológicas observadas.

Para o exame histopatológico foram colhidas as seguintes porções do SNC: córtex frontal direito e esquerdo, córtex parie- tal direito e esquerdo, córtex temporal direito e esquerdo, córtex occipital direito e esquerdo, núcleos da base direito e esquerdo, tálamo direito e esquerdo, colículo rostral do mesencéfalo, colículo caudal, incluindo o leminisco lateral, ponte, bulbo, cerebelo e medula espinhal. As amostras foram fixadas em formol tamponado a $10 \%$, incluídas em parafina e seccionadas para a confecção das lâminas histológicas coradas com Hematoxilina e Eosina.

No exame histopatológico, utilizou-se a classificação sugerida por Sant'ana et al. (2009) e Cunha et al. (2011). Foram avaliadas a presença de necrose neuronal, gliose, edema, tumefação endotelial, congestão, hemorragia, neuronofagia, manguitos perivasculares, malácia e infiltrado inflamatório. Todas as lâminas foram avaliadas por três patologistas com experiência em neuropatologia, sem conhecimento prévio sobre a qual grupo pertenciam os animais avaliados.

Foram colhidos soro (M1 e M12) e um fragmento de fígado (necropsia), de cada animal para dosagem de cobre ${ }^{19}$.

Os procedimentos realizados foram previamente aprovados pela Câmara de Ética em Experimentação Animal da FMVZ/ UNESP/Botucatu (Protocolo nำ127/2009-CEUA).

\section{Análise estatística}

Os resultados de frequência cardíaca, frequência respiratória e pH ruminal foram avaliados por análise de variância (ANOVA), seguida pelo teste de Tukey. Os resultados dos movimentos ruminais e da dosagem de sulfeto de hidrogênio ruminal foram avaliados pelo teste Wilcoxon para comparações feitas dentro de um mesmo grupo e pelo teste de Mann Whitney para comparações entre os grupos. Os valores de $\mathrm{H}_{2} \mathrm{~S}$ ruminal inferiores a $25 \mathrm{ppm}$ foram considerados como 24 para a realização da análise estatística. Os valores de cobre sérico nos dois momentos aferidos foram comparados dentro do mesmo grupo pelo teste $t$ de Student pareado. Os valores do cobre sérico no mesmo momento entre os grupos e os valores do cobre hepático foram comparados pelo teste t de Student não pareado(GraphPad Software). A diferença estatística foi considerada para $\mathrm{p} \leq 0,05$ (Sampaio 2010).

\section{RESULTADOS}

A ração e o feno fornecidos na dieta antes da adição do sulfato de sódio continham $0,2 \%$ e $0,26 \%$ de enxofre na matéria seca, respectivamente, e a água continha $8 \mathrm{mg} / \mathrm{L} \mathrm{de}$ enxofre.

As análises bromatológicas revelaram os seguintes resultados: feno de Coast-cross (Cynodon dactylon) com $89,3 \%$ de matéria seca, $10,3 \%$ de proteína bruta, $6,2 \%$ de minerais, $81,8 \%$ de fibra em detergente neutro, $40,3 \%$ de fibra em detergente ácido, 4,6\% de lignina e 33,5\% de celu-

\footnotetext{
${ }^{12}$ Bomba modelo AP20S, Sensidyne ${ }^{\circledR}$ Sensidyne Industrial Health and Safety Instrumentation, Clearwater, Florida, USA.

${ }^{13}$ Tubos colorimétricos modelo 120 SF, Sensidyne ${ }^{\circledR}$ Sensidyne Industrial Health and Safety Instrumentation, Clearwater, Florida, USA.

${ }^{14}$ Tubos colorimétricos modelo 120 SH, Sensidyne ${ }^{\circ}$ Sensidyne Industrial Health and Safety Instrumentation, Clearwater, Florida, USA.

${ }^{15}$ I-Stat 1 Analyzer ${ }^{\circledR}$, Abbot Point of Care Inc., East Windsor, New Jersey, USA.

${ }^{16}$ I-Stat EG7+®, Abbot Point of Care Inc., East Windsor, New Jersey, USA.

${ }^{17}$ Phtek ${ }^{\circledR}$, modelo pH100, Tecnoglobo Equipamentos para Laboratório, Curitiba, PR.

${ }^{18}$ Centro de Diagnóstico por Imagem da FMVZ, Unesp/ Botucatu. Aparelho, Shimadsu ${ }^{\circledR}$, modelo SCT 7800 TC.

${ }^{19}$ Centro de Assistência Toxicológica (CEATOX) do Instituto de Biociências da Unesp, Botucatu, utilizando-se a espectrometria por absorção atômica no aparelho GBC - Scientific Equipment Pty Ltda, modelo 932 AA.
} 
lose; ração experimental 89,7\% de matéria seca, 16,9\% de proteína bruta, 5,0\% de extrato etéreo, $12,6 \%$ de minerais, $20,7 \%$ de fibra em detergente neutro, 10,4\% de fibra em detergente ácido, 5,1\% de lignina e 7,7\% de celulose.

Os teores de minerais, em $\mathrm{g} / \mathrm{kg}$ de MS ou $\mathrm{mg} / \mathrm{kg}$ de MS, encontrados no feno foram $1 \mathrm{~g}$ de $\mathrm{P}, 5 \mathrm{~g}$ de $\mathrm{K}, 3 \mathrm{~g} \mathrm{Ca}, 1,9 \mathrm{~g}$ de $\mathrm{Mg}, 9 \mathrm{~g}$ de $\mathrm{B}, 6 \mathrm{mg}$ de $\mathrm{Cu}, 189 \mathrm{mg}$ Fe, 383mg de $\mathrm{Mn}$ e $35 \mathrm{mg}$ $\mathrm{Zn}$. Este feno continha $13 \mathrm{~g} / \mathrm{kg}$ de nitrogênio. Na ração encontraram-se $5,3 \mathrm{~g} / \mathrm{kg}$ de fósforo, $5,9 \mathrm{~g} / \mathrm{kg}$ de cálcio, $3 \mathrm{~g} / \mathrm{kg}$ de magnésio, 7,5g/kg de boro, $15 \mathrm{mg} / \mathrm{kg}$ de cobre, $480 \mathrm{mg} /$ $\mathrm{kg}$ de ferro, $116,5 \mathrm{mg} / \mathrm{kg}$ de manganês, $146 \mathrm{mg} / \mathrm{kg}$ de zinco e 5,75mg/kg de molibdênio. 0 teste de HPLC realizado não detectou tiamina na ração.

A quantidade de ração consumida pelos ovinos nos três grupos variou entre 3 e $3,6 \%$ do peso vivo durante todo o período experimental. 0 ganho de peso médio diário durante as três fases do experimento em gramas por dia foi: 46g (G1), 40g (G2) e 33g (G3).

No grupo controle um animal morreu no sexagésimo segundo dia da fase 3, após desenvolver urolitíase. No exame físico, entre M4 e M12, quatro animais do grupo G1 e todos os animais dos grupos G2 e G3 apresentaram focos de crepitação grossa na auscultação pulmonar. Durante o exame físico era possível sentir, a partir do M3, o odor de $\mathrm{H}_{2} \mathrm{~S}$ exalado pelos animais dos grupos 2 e 3 . Os ovinos dos grupos que receberam alto teor de enxofre na dieta apresentaram episódios recorrentes de diarreia a partir do M3, sendo que, nos animais do G3, esses episódios foram mais frequentes. Nenhum animal apresentou sinais clínicos de PEM durante o experimento.

A média e o desvio padrão em cada fase da frequência cardíaca em batimentos por minuto e frequência respiratória em movimentos por minuto (FR mpm) para cada grupo estão apresentados no quadro 1. Não houve diferença estatística entre os grupos ( $\mathrm{p}>0,05)$.

Quadro 1. Valores médios e desvios-padrão da frequência cardíaca em batimentos por minuto e frequência respiratória em movimentos por minuto dos ovinos nos 12 diferentes momentos aferidos durante as três fases experimentais

\begin{tabular}{|c|c|c|c|c|c|c|}
\hline & \multicolumn{3}{|c|}{ Frequência cardíaca } & \multicolumn{3}{|c|}{ Frequência Respiratória } \\
\hline & G1 & G2 & G3 & G1 & G2 & G3 \\
\hline \multicolumn{7}{|c|}{ Fase 1} \\
\hline M1 & $89 \pm 19$ & $99 \pm 15$ & $82 \pm 20$ & $27 \pm 8$ & $29 \pm 16$ & $26 \pm 4$ \\
\hline M2 & $82 \pm 14$ & $79 \pm 16$ & $78 \pm 12$ & $23 \pm 5$ & $23 \pm 5$ & $25 \pm 5$ \\
\hline \multicolumn{7}{|c|}{ Fase 2} \\
\hline M3 & $87 \pm 20$ & $93 \pm 13$ & $89 \pm 26$ & $41 \pm 19$ & $44 \pm 17$ & $42 \pm 21$ \\
\hline \multicolumn{7}{|c|}{ Fase 3} \\
\hline M4 & $117 \pm 27$ & $112 \pm 9$ & $105 \pm 19$ & $37 \pm 22$ & $39 \pm 11$ & $26 \pm 7$ \\
\hline M5 & $126 \pm 20$ & $115 \pm 13$ & $95 \pm 28$ & $48 \pm 25$ & $51 \pm 24$ & $49 \pm 35$ \\
\hline M6 & $119 \pm 22$ & $118 \pm 19$ & $103 \pm 17$ & $41 \pm 30$ & $29 \pm 07$ & $35 \pm 4$ \\
\hline M7 & $119 \pm 14$ & $104 \pm 15$ & $98 \pm 20$ & $43 \pm 38$ & $62 \pm 35$ & $35 \pm 14$ \\
\hline M8 & $115 \pm 20$ & $114 \pm 16$ & $115 \pm 11$ & $46 \pm 27$ & $52 \pm 35$ & $47 \pm 31$ \\
\hline M9 & $103 \pm 14$ & $116 \pm 8$ & $96 \pm 15$ & $42 \pm 23$ & $38 \pm 16$ & $47 \pm 28$ \\
\hline M10 & $110 \pm 24$ & $104 \pm 22$ & $98 \pm 22$ & $34 \pm 17$ & $57 \pm 49$ & $39 \pm 19$ \\
\hline M11 & $109 \pm 15$ & $112 \pm 21$ & $101 \pm 15$ & $30 \pm 17$ & $37 \pm 15$ & $44 \pm 37$ \\
\hline M12 & $114 \pm 13$ & $106 \pm 7$ & $99 \pm 17$ & $33 \pm 7$ & $45 \pm 37$ & $40 \pm 21$ \\
\hline
\end{tabular}

Grupos: G1 = 0,2\% enxofre MS; G2 = 0,9\% enxofre MS; G3 = 1,2\% enxofre MS. Fase 1: adaptação (M1 = $1^{\circ}$ dia; $M 2=14^{\circ}$ dia). Fase 2: transição (M3 = $7^{\circ}$ dia). Fase 3: fornecimento dieta com $95 \%$ da ração experimental e $5 \%$ de feno $\left(M 4=10^{\circ}\right.$ dia; $M 5=20^{\circ}$ dia; M6 = 30 ${ }^{\circ}$ dia; $M 7=40^{\circ}$ dia; $M 8=50^{\circ}$

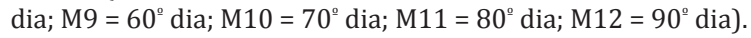

Quadro 2. Valores médios e desvios-padrão do sulfeto de hidrogênio ruminal em ppm avaliados nos ovinos durante as três fases do protocolo de indução de polioencefalomalacia

\begin{tabular}{lccc} 
& G1 & G2 & G3 \\
\hline Fase 1 & & & \\
M1 & $121 \pm 107$ & $314 \pm 267$ & $216 \pm 385$ \\
M2 & $376 \pm 434$ & $188 \pm 244$ & $233 \pm 184$ \\
Média & $\mathbf{2 4 9} \pm \mathbf{3 2 9} \mathbf{A a}$ & $\mathbf{2 5 1} \pm \mathbf{2 5 3} \mathbf{A a}$ & $\mathbf{2 2 5} \pm \mathbf{2 8 8} \mathbf{A a}$ \\
Fase 2 & & & \\
M3 & $62 \pm 31$ & $3442 \pm 2752$ & $2650 \pm 2162$ \\
Média & $\mathbf{6 2} \pm \mathbf{3 1} \mathbf{A a}$ & $\mathbf{3 4 4 2} \pm \mathbf{2 7 5 2} \mathbf{B b}$ & $\mathbf{2 6 5 0} \pm \mathbf{2 1 6 2} \mathbf{B b}$ \\
Fase 3 & & & \\
M4 & $137 \pm 107$ & $2283 \pm 884$ & $4150 \pm 2155$ \\
M5 & $91 \pm 152$ & $2967 \pm 1368$ & $3633 \pm 1693$ \\
M6 & $61 \pm 79$ & $4450 \pm 1677$ & $3750 \pm 2770$ \\
M7 & $71 \pm 76$ & $2633 \pm 1606$ & $4367 \pm 1571$ \\
M8 & $48 \pm 37$ & $4900 \pm 219$ & $4400 \pm 1490$ \\
M9 & $54 \pm 44$ & $4467 \pm 2066$ & $4883 \pm 1280$ \\
M10 & $24 \pm 0$ & $3800 \pm 1442$ & $5000 \pm 1517$ \\
M11 & $24 \pm 0$ & $2267 \pm 1491$ & $3533 \pm 1986$ \\
M12 & $24 \pm 0$ & $2633 \pm 1244$ & $3850 \pm 1985$ \\
Média & $\mathbf{6 4} \pm \mathbf{8 0} \mathbf{A a}$ & $\mathbf{3 3 7 8} \pm \mathbf{1 6 3 8} \mathbf{B b}$ & $\mathbf{4 1 7 1} \pm \mathbf{1 8 0 2} \mathbf{B b}$
\end{tabular}

Grupos: G1 = 0,2\% enxofre MS; G2 = 0,9\% enxofre MS; G3 = 1,2\% enxofre MS. Fase 1: adaptação (M1 = $1^{\circ}$ dia; M2 = 14º dia). Fase 2: transição (M3 $=7^{\circ}$ dia). Fase 3: fornecimento dieta com 95\% da ração experimental e

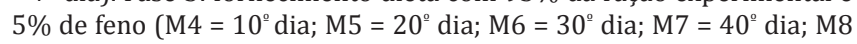

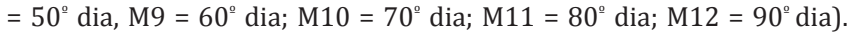
Letras maiúsculas diferentes nas colunas indicam diferença significativa $(\mathrm{p}<0,05)$ entre as fases do período experimental e letras minúsculas diferentes indicam diferença significativa $(p<0,05)$ entre os grupos.

Não houve diferença estatística entre os grupos quanto aos valores médios de temperatura retal aferidos em cada momento. Na fase 3 a frequência dos movimentos ruminais nos animais dos grupos G2 e G3 foi estatisticamente inferior a dos animais do grupo $\mathrm{G} 1(\mathrm{p}<0,05)$.

Nenhum animal apresentou quadro clínico de acidose metabólica. 0 pH sanguíneo nas fases 2 e 3 apresentou aumento significativo em relação à fase 1 nos três grupos $(\mathrm{p}<0,05)$. Não houve diferença estatística $(\mathrm{p}>0,05)$ entre os grupos com relação à avaliação de $\mathrm{pH}$ sanguíneo, $\mathrm{PCO}_{2}, \mathrm{PO}_{2}$, $\mathrm{BE}, \mathrm{HCO}_{3}{ }^{-}, \mathrm{tCO}_{2}, \mathrm{sO}_{2}, \mathrm{Na}^{+}, \mathrm{K}^{+}, \mathrm{iCa}^{2+}$, Hct e $\mathrm{Hb}$.

As médias e os desvios padrão do $\mathrm{pH}$ ruminal aferidos em cada fase foram as seguintes: fase $1, \mathrm{G} 1(7,19 \pm 0,30), \mathrm{G} 2$ $(7,06 \pm 0,40)$ e G3 $(7,11 \pm 0,14)$; fase 2 , G1 $(6,61 \pm 0,14), \mathrm{G} 2$ $(6,63 \pm 0,21)$ e G3 $(6,81 \pm 0,44)$; fase 3 G1 $(6,43 \pm 0,27)$, G2 $(6,20 \pm 0,27)$ e G3 $(6,17 \pm 0,26)$. Não houve diferença estatística nos valores do $\mathrm{pH}$ ruminal entre os grupos durante as fases experimentais ( $\mathrm{p}>0,05)$.

Os valores de $\mathrm{H}_{2} \mathrm{~S}$ ruminal no grupo controle não mostraram diferença estatística quando comparados com as fases 1, 2 e 3. Nos ovinos dos grupos G2 e G3 observou-se diferença significativa $(\mathrm{p}<0,05)$ entre as fases 1 e 2 e fase 1 e 3. Quando a comparação foi feita entre os grupos G1 e G2 e G1 e G3, houve diferença significativa nas fases 2 e 3 $(\mathrm{p}<0,05)$. Já, na comparação feita entre os grupos G2 e G3, não houve diferença estatística ( $\mathrm{p}>0,05)$. Os valores de $\mathrm{H}_{2} \mathrm{~S}$ aferidos nas fases 2 e 3 foram cerca de 50 vezes maiores no G2 e no G3, em relação às mesmas fases do grupo controle (Quadro 2).

No exame de tomografia computadorizada contrastada, nenhuma alteração foi observada no encéfalo dos animais. 
Os resultados das dosagens de cobre sérico e hepático estão apresentados no Quadro 3. Apesar de não haver diferença estatística, quando a comparação entre o M1 e M12 foi realizada dentro de cada grupo, ocorreu uma queda nos níveis séricos de cobre no M12 (G1: 11,47\%, G2: 21,90\% e G3: $36,88 \%$ ). Quanto maior a ingestão de enxofre menores foram os níveis hepáticos de cobre detectados, sendo estatisticamente diferente $(\mathrm{p}<0,05)$ quando comparados o G1 e G2, G1 e G3 e G2 e G3.

\section{Quadro 3. Valores médios e desvio padrão da dosagem de cobre sérico $(\mu / \mathrm{mL})$ avaliados nos momentos M1 e M12 e hepático (ppm) no M12}

\begin{tabular}{lccc}
\hline & G1 & G2 & G3 \\
\hline Soro M1 & $1,19 \pm 0,22 \mathrm{Aa}$ & $1,23 \pm 0,27 \mathrm{Aa}$ & $0,99 \pm 0,35 \mathrm{Aa}$ \\
Soro M12 & $1,06 \pm 0,30 \mathrm{Aa}$ & $0,96 \pm 0,45 \mathrm{Aa}$ & $0,62 \pm 0,07 \mathrm{Ab}$ \\
Fígado & $197,49 \pm 52,06 \mathrm{a}$ & $135,18 \pm 26,62 \mathrm{~b}$ & $74,86 \pm 18,42 \mathrm{c}$
\end{tabular}

Grupos: G1 = 0,2\% enxofre M; G2 = 0,9\% enxofre MS; G3 = 1,2\% enxofre MS. M1: $1^{\circ}$ dia da fase de adaptação. M12 = 90ำ dia da fase 3 (fornecimento dieta com $95 \%$ da ração experimental e 5\% de feno). Letras maiúsculas diferentes nas colunas indicam diferença significativa $(\mathrm{p}<0,05)$ entre os momentos dentro do mesmo grupo e letras minúsculas diferentes indicam diferença significativa $(\mathrm{p}<0,05)$ entre os grupos em cada momento.

Na necropsia não foram observadas lesões encefálicas sugestivas de PEM. Quatro animais do G1 e todos os animais dos grupos 2 e 3 apresentaram áreas multifocais a coalescentes de coloração avermelhada ou acinzentada, consistência aumentada na região cranioventral dos pulmões, compatíveis com broncopneumonia nas fases aguda e crônica, respectivamente. Estas regiões foram negativas no teste de docimasia. A extensão e a gravidade da pneumonia foram maiores nos animais dos grupos 2 e 3 , sendo que cinco animais do G2 e cinco animais do G3 apresentaram áreas de consolidação do parênquima pulmonar, enquanto que nos animais do grupo controle apenas discretas áreas multifocais de pneumonia foram detectadas cranioventralmente. A mucosa do intestino delgado de dois animais do G2 e três animais do G3 estava avermelhada e com conteúdo líquido amarelado. Microscopicamente, as áreas avermelhadas do pulmão dos animais apresentaram hiperemia e edema acentuados, discreta presença de neutrófilos e raros macrófagos; enquanto as áreas acinzentadas apresentaram proliferação de tecido conjuntivo, infiltrado inflamatório composto por neutrófilos e macrófagos, hiperplasia do tecido linfoide associado a brônquios, bronquiectasia, atelectasia e enfisema, caracterizando um quadro de broncopneumonia supurativa crônica acentuada. No intestino delgado de dois animais do G2 e três do G3 havia enterite discreta a moderada, com necrose do ápice das vilosidades, infiltrado inflamatório composto predominantemente por neutrófilos e menor quantidade de linfócitos e plasmócitos, presença de fibrina e aglomerados multifocais de substância basofílica finamente granular compatíveis com colônias bacterianas. No exame histopatológico das 17 regiões do encéfalo, colhidas de cada animal, não foram observadas lesões compatíveis com PEM.

\section{DISCUSSÃO}

Os resultados das análises bromatológica e mineral da ração fornecida aos ovinos foram semelhantes aos obtidos por Sager et al. (1990) e Cunha et al. (2011), que utilizaram ração com os mesmos ingredientes e conseguiram induzir PEM em bovinos pelo consumo excessivo de enxofre após 15 e 6 dias, respectivamente.

A quantidade de ração consumida pelos ovinos nos três grupos variou entre 3 e 3,6\% do peso vivo de MS durante todo o período experimental, quantidade adequada para ovinos de quatro a sete meses de idade (NRC 2007). Porém, o ganho de peso dos animais não foi satisfatório em nenhum grupo; quanto maior a ingestão de enxofre, menor foi o ganho de peso médio diário, concordando com Alves de Oliveira et al. (1996). Os referidos autores forneceram dieta à base de feno e concentrado a dois grupos de ovinos, um com ingestão de alto teor de enxofre $(0,63 \%)$ na MS e o outro com $0,2 \%$ de enxofre na MS; no grupo que ingeriu $0,63 \%$ de enxofre registrou-se menor ganho de peso. Kung Jr (2008) também observou que o excesso de enxofre na dieta diminui o ganho de peso dos animais.

0 confinamento pode predispor os ovinos para doenças respiratórias (Martin, 1996). Aumento da frequência respiratória e lesões pulmonares são comuns em ruminantes com ingestão de alto teor de enxofre (Bulgin et al. 1990, Rousseaux et al. 1991, Krasicka et al. 1999, Cunha et al. 2011). Outro fator, que pode ter favorecido a ocorrência de doença respiratória, é a granulometria da ração, pois a forma farelada pode causar irritação nas vias aéreas (Silva et al. 2007). A taquipneia observada nos três grupos a partir do M3 poderia estar relacionada à broncopneumonia apresentada por quatro animais do G1 e por todos os animais dos grupos G2 e G3 durante as fases 2 e 3. As lesões pulmonares mais acentuadas nos ovinos dos grupos G2 e G3 podem ser explicadas pela lesão direta do $\mathrm{H}_{2} \mathrm{~S}$ ao tecido pulmonar (Kung Jr 2008), pois até $60 \%$ do $\mathrm{H}_{2} \mathrm{~S}$ eructado pode ser inalado pelo animal (Bulgin et al. 1996).

0 alto teor de enxofre na dieta pode provocar distúrbios digestórios (Bulgin et al. 1996) como a diarreia observada nos animais dos grupos G2 e G3. A maior frequência dos episódios de diarreia observados nos ovinos do G3 pode ser explicada pela ação laxativa do sulfato de sódio (Ramos et al. 1988) e a quantidade de sulfato de sódio ingerida pelos animais do G3 foi maior do que a ingerida pelos animais do G2. Enterite, como observada nos animais dos grupos 2 e 3, já foi descrita em ovinos (White 1964) e bovinos (Gunn et al. 1987) consumindo dieta com alto teor de enxofre.

A acidose metabólica é um dos fatores responsáveis por desencadear PEM por deficiência de tiamina em ruminantes (Gould 2000), razão para a realização da hemogasometria venosa e da aferiçãodo $\mathrm{pH}$ ruminal. Nos 12 momentos aferidos, tanto o $\mathrm{pH}$ sanguíneo quanto o $\mathrm{pH}$ ruminal mantiveram-se dentro dos valores de referência considerados normais para a espécie (Radostits et al. 2007). Neste trabalho, além da substituição gradativa do feno pela ração (fase 2), havia $2 \%$ bicarbonato de sódio na dieta o que, segundo Damir et al. (1990), aumenta o pH sanguíneo, além de ajudar na prevenção da acidose láctica ruminal e, consequentemente, a acidose metabólica.

Quando se comparou $\mathrm{pH}$ sanguíneo, $\mathrm{PCO}_{2}, \mathrm{PO}_{2}, \mathrm{BE}$, $\mathrm{HCO}_{3}^{-}, \mathrm{tCO}_{2}, \mathrm{SO}_{2}, \mathrm{Na}^{+}, \mathrm{K}^{+}, \mathrm{iCa}^{2+}$ entre os grupos em um mesmo momento não houve diferença estatística, isso demons- 
tra que o alto teor de enxofre na dieta não interferiu nestas variáveis.

Em bovinos o odor de $\mathrm{H}_{2} \mathrm{~S}$ exalado pelos animais foi associado com o início dos sinais clínicos de PEM (Sager et al. 1990, Gould et al. 1997). No presente estudo, apesar de nenhum animal ter desenvolvido sinais clínicos do M3 até o M12 era possível sentir odor de $\mathrm{H}_{2} \mathrm{~S}$ exalado pelos animais dos grupos G2 e G3.

$\mathrm{O}$ elevado teor de $\mathrm{H}_{2} \mathrm{~S}$ detectado nos animais dos grupos G2 e G3 a partir da fase 2 demonstrou que a técnica de dosagem de $\mathrm{H}_{2} \mathrm{~S}$ com utilização de tubos colorimétricos também é eficiente na espécie ovina para identificar rebanhos que estejam consumindo alto teor de enxofre na dieta. No entanto, apesar dos níveis de $\mathrm{H}_{2} \mathrm{~S}$ detectados estarem elevados, nenhum animal desenvolveu quadro clínico de PEM, corroborando com Neville et al. (2010). A técnica de dosagem de $\mathrm{H}_{2} \mathrm{~S}$ ruminal foi desenvolvida por Gould et al. (1997) e outros autores comprovaram a eficácia do teste para identificar rebanhos de bovinos com grande quantidade de enxofre na dieta (Loneragan et al. 1998, Cunha et al. 2010, Cunha et al. 2011). Segundo Burgess (2008), valores de $\mathrm{H}_{2} \mathrm{~S}$ ruminal acima de 500ppm são tóxicos para bovinos; para ovinos este valor ainda não foi estabelecido. Em bovinos, após o aumento do $\mathrm{H}_{2} \mathrm{~S}$ ruminal os animais desenvolveram PEM (Gould et al. 1997), portanto, é possível que bovinos e ovinos tenham tolerâncias diferentes aos níveis de $\mathrm{H}_{2} \mathrm{~S}$ no rúmen.

$\mathrm{A}$ alta concentração de $\mathrm{H}_{2} \mathrm{~S}$ no rúmen pode diminuir a motricidade ruminal (Kandylis 1984), o que explica o número reduzido de movimentos ruminais nos animais dos grupos G2 e G3 em relação ao grupo G1.

O enxofre é um importante antagonista do cobre (Humphries et al. 1983) e isto pode ser evidenciado pelas dosagens de cobre realizadas no soro dos animais no M1 e M12 e no fígado no M12 (Quadro 3). Quanto mais enxofre na dieta menores foram os níveis de cobre detectados nos grupos. 0 fato dos níveis de cobre sérico não serem significativamente menores no M12 em relação ao M1 pode ser explicado pelo fato do fígado ter capacidade de armazenar cobre; primeiro os níveis hepáticos de cobre diminuem para depois essa queda ser refletida do soro (Blakley \& Hamilton 1985). No M12 no grupo 3, tanto os níveis de cobre sérico quanto hepático estavam abaixo do valor mínimo considerado normal para a espécie ovina, que é de $0,7 \mu \mathrm{g} /$ $\mathrm{ml}$ no soro e 88 ppm no fígado (Pugh 2004).

Os minerais como cobre, zinco, ferro e molibdênio podem interferir na toxicose por enxofre (Gould 1998). 0 molibdênio, o cobre e o enxofre podem se ligar formando o cobre tiomolibdato, que é insolúvel e não utilizável (Vásquez et al. 2001), assim como o cobre, o zinco e o ferro também formam sais insolúveis com o sulfito (Suttle 1991). Portanto, animais que ingerem dietas com alto teor de enxofre necessitam de uma quantidade maior dos outros minerais (Gould 1998). Dieta com baixos níveis de cobre também ficam mais suscetíveis à PEM por excesso de enxofre (Low et al. 1996). Na ração fornecida, os níveis de cobre, molibdênio, ferro e zinco estavam dentro dos níveis recomendados para ovinos (NRC 2005) e somente os níveis de enxofre diferia entre as rações, o que deixa claro que o enxofre foi o responsável pela queda nos níveis de cobre sérico e hepático.

0 intuito de realizar o exame de tomografia computadorizada nos ovinos foi verificar se durante a apresentação dos sinais clínicos de PEM, era possível identificar através da imagem o local da lesão no encéfalo e correlacionar esse achado com os sinais clínicos apresentados. Porém nenhum ovino desenvolveu sinais clínicos da doença e nenhuma lesão foi observada nos animais submetidos ao exame.

0 exame de tomografia computadorizada pode auxiliar no diagnóstico de doenças neurológicas; a técnica já foi empregada com sucesso no diagnóstico cenurose em ovinos (Gonzalo-Orden et al. 1999). Por outro lado, o exame de ressonância magnética (RM) pode identificar diferentes estruturas anatômicas no encéfalo com maior precisão e permite a detecção de alterações mínimas (Amaro Júnior \& Yamashita 2001). A RM tem auxiliado no diagnóstico de PEM em bezerros (Tsuka et al. 2008), mas infelizmente este exame ainda não está disponível na instituição onde a presente pesquisa foi realizada.

Os animais dos grupos G2 e G3 consumiram elevado teor de enxofre na dieta, produziram grande quantidade de $\mathrm{H}_{2} \mathrm{~S}$ no rúmen, porém não apresentaram sinais clínicos de PEM. Resultados semelhantes foram descritos por Neville et al. (2010) em dois experimentos. No primeiro experimento, os autores utilizaram 240 ovinos divididos em quatro grupos de 60 animais. Esses ovinos receberam dieta à base de restos de grãos de destilaria, ricos em enxofre $(0,73 \%)$, por 110 dias. 0 grupo 1 serviu como controle e os outros grupos foram suplementados com tiamina (grupo $2,50 \mathrm{mg} /$ animal/dia; grupo $3,100 \mathrm{mg} /$ animal/dia; e grupo 4, 150mg/animal/dia). Nenhum animal do experimento apresentou sinais clínicos de PEM. Em um segundo estudo, Neville et al. (2010) utilizaram 55 animais divididos em cinco grupos. Quatro grupos receberam tratamentos iguais ao do primeiro estudo e o grupo 5 recebeu dieta com $0,87 \%$ de enxofre e foram suplementados com $150 \mathrm{mg} / \mathrm{animal} /$ dia de tiamina. 0 segundo estudo durou 112 dias e nenhum animal desenvolveu PEM. Neste segundo trabalho realizou-se exame histopatológico do encéfalo dos animais e nenhuma alteração de PEM foi observada, apesar dos autores terem detectado elevado teor de $\mathrm{H}_{2} \mathrm{~S}$ ruminal. Além disso, Edwin et al. (1968) também não conseguiram induzir PEM em um ovino ingerindo 9 gramas por dia de sulfato de sódio durante um ano.

Em contrapartida, outros autores conseguiram induzir PEM em ovinos fornecendo dieta rica em carboidratos e com níveis de enxofre inferiores aos utilizados na presente pesquisa. Gooneratne et al. (1989) induziram PEM em três ovinos que consumiram dieta à base de cevada, farelo de soja e alfafa com $0,63 \%$ de enxofre na MS; o experimento durou 12 semanas e na segunda e na terceira semanas os animais apresentaram sinais clínicos de PEM. Rousseaux et al. (1991) induziram PEM em sete de 22 ovinos que consumiram ração contendo $59 \%$ de cevada, $5 \%$ de farelo de soja e $36 \%$ de alfafa, com $0,63 \%$ de enxofre na MS durante 14 semanas, os animais apresentaram sinais clínicos entre a terceira e a sétima semana. Alves de Oliveira et al. (1996) forneceram uma dieta com $67 \%$ de concentrado e $33 \%$ de 
volumoso, com 0,6\% de enxofre na MS durante 12 semanas a quatro ovinos e um animal apresentou sinais clínicos de PEM na sétima semana. Krasicka et al. (1999) forneceram a cinco ovinos uma dieta com $79 \%$ de concentrado e $21 \%$ de volumoso, com $0,8 \%$ de enxofre na MS durante 12 semanas e todos os animais apresentaram sinais clínicos de PEM na última semana do experimento.

A opção pela utilização do sulfato de sódio como fonte de enxofre no presente trabalho foi baseado nos resultados descritos na literatura; Alves de Oliveira et al. (1996) utilizaram sulfato de sódio $(0,2 \%)$ associado a sulfato de amônio (4\%) e Krasicka et al. (1999) utilizaram sulfato de sódio $(0,8 \%)$; em ambos os experimentos os ovinos desenvolveram PEM. Em bovinos o sulfato de sódio também tem sido utilizado com sucesso para a indução da doença (Gould et al. 1997, Cunha et al. 2011). Outro modelo experimental utilizado é o fornecimento de hidrossulfeto de sódio via sonda esofágica, este protocolo induziu PEM em ovinos poucas horas após a administração, porém a utilização do hidrossulfeto de sódio não é recomendada devido a sua alta toxicidade, também para humanos (McAllister et al. 1992).

Nos surtos de PEM induzidos por enxofre em ovinos, a morbidade variou entre 12 e 91\% (Jefreyet al. 1989, Bulgin et al. 1996, Low et al. 1996, Lima et al. 2005) enquanto que, experimentalmente, a morbidade variou entre 0 e $100 \%$ (Gooneratne et al. 1989, Rousseaux et al. 1991, Alves de Oliveira et al. 1996, Krasicka et al. 1999, Neville et al. 2010). Isto pode indicar a existência de suscetibilidade individual dos animais em resposta a altos níveis de enxofre na dieta. Além disso, a exigência de enxofre é maior em ovinos do que em bovinos (NRC 2005), portanto, os ovinos podem ser mais resistentes que os bovinos a teores elevados de enxofre.

Vale ressaltar que não foi detectada tiamina na ração, o que foi importante, pois ovinos ingerindo alto teor de enxofre na dieta podem sofrer lesões no SNC e não apresentar sinais clínicos de PEM quando suplementados com tiamina (Gooneratne et al. 1989, Rousseaux et al. 1991, Olkowski et al. 1992).

Na PEM os danos estruturais no cérebro nem sempre se manifestam como um efeito funcional; os animais podem estar acometidos e não apresentarem sinais clínicos (Gooneratneet al. 1989, Rousseaux et al. 1991, Olkowski et al. 1992). Portanto, somente após o exame histopatológico do encéfalo de todos os animais revelar ausência de alterações de PEM, foi possível afirmar que a dieta com alto teor de enxofre, fornecida nas condições descritas neste experimento, não foi capaz de induzir PEM nos ovinos, resultados que corroboram os obtidos por Neville et al. (2010).

\section{CONCLUSÕES}

0 protocolo utilizado neste estudo com base em dieta contendo $0,9 \%$ e $1,2 \%$, de enxofre não induziu PEM, em 111 dias de exposição a essa situação, apesar do alto teor de $\mathrm{H}_{2} \mathrm{~S}$ ruminal detectado.

Recomenda-se a utilização da técnica de dosagem de $\mathrm{H}_{2} \mathrm{~S}$ ruminal também na espécie ovina para identificar rebanhos com ingestão de alto teor de enxofre na dieta.

A participação de fatores ainda não identificados, asso- ciados ou não ao consumo excessivo de enxofre, constitui uma vasta área de estudos no que diz respeito a PEM em ovinos.

Agradecimentos.- À Fundação de Apoio à Pesquisa do Estado de São Paulo pelo suporte financeiro em forma de bolsa de estudo ao primeiro autor e auxílio regular à pesquisa (Processos 2009/12500-2 e 2010/01684-2, respectivamente).

\section{REFERÊNCIAS}

Alves de Oliveira L., Blain C.J., Corso V.B., Durix A. \& Komisarczuk Bony S. 1996. Effect of a high sulfur diet on rumen microbial activity and rumen thiamine status in sheep receiving a semi-synthetic, thiamine-free diet. Reprod. Nutr. Dev. 36:31-42.

Amaro Júnior E. \& Yamashita H. 2001. Aspectos básicos de tomografia computadorizada e ressonância magnética. Revta Bras. Psiquiatr. 23:12.

Blakley B.R. \& Hamilton D.L. 1985. Ceruloplasmin as an indicator of copper status in cattle and sheep. Can. J. Comp. Med. 49:405-408.

Bulgin M.S., Stuart S.D. \& Mather G. 1996. Elemental sulfur toxicosis in a flock of sheep. J. Am. Vet. Med. Assoc. 208:1063-1065.

Burgess B.A. 2008. Polioencephalomalacia. Large Anim. Vet. 8:1-6.

Cunha P.H.J., Badial P.R., Cagnini D.Q., Oliveira-Filho J.P., Moares L.F., Takahira R.K., Amorim R.L. \& Borges A.S. 2011. Polioencefalomalacia experimental em bovinos induzida por toxicose por enxofre. Pesq. Vet. Bras. 31(1):41-52.

Cunha P.H.J., Bandarra P.M., Dias M.M., Borges A.S. \& Driemeier D. 2010. Surto de polioencefalomalacia por ingestão excessiva de enxofre na dieta em bezerros no Rio Grande do Sul. Pesq. Vet. Bras.. 30(8):613-617.

Damir H.A., Scott D., Thomson J.K., Topps J.H., Buchan W. \& Pennie K. 1990. The effect of a change in blood acid-base on body composition and mineral retention in growing lambs. Anim. Prod. 51:527-534.

De Lahunta A. 2009. Veterinary Neuroanatomy and Clinical Neurology. $3^{\text {rd }}$ Ed. Saunders Elsevier, St Louis, 515p.

Dirksen G., Gründer H.D. \& Stöber M. 1993. Exame Clínico dos Bovinos. 3ạ ed. Guanabara Koogan, Rio de Janeiro, p.373-381.

Drewnoski M.E., Ensley S.M., Loy D.D., Imerman P.M., Rathje J.A. \& Hansen S.L. 2012. Assessment of ruminal hydrogen sulfide or urine thiosulfate as diagnostic tools for sulfur-induced polioencephalomalacia in cattle. J. Vet. Diagn. Invest. 24(4):702-709.

Edwin E.E., Lewis G. \& Allcroft R. 1968.Cerebrocortical necrosis: A hypothesis for the possible role of thiaminases in its pathogenesis. Vet. Rec. 83:175-176

Feitosa F.L.F. 2004. Semiologia Veterinária: a arte do diagnóstico. Roca, São Paulo. 807p.

Gonzalo-Orden J.M., Díez A., Altonága J.R., Gonzalo J.M. \& Orden M.A. 1999. Computed tomographic findings in ovine coenurosis. Vet. Radiol. Ultrasound 40(5):441-444.

Gooneratne S.R., Olkowski A.A. \& Christensen D.A. 1989. Sulfur-induced polioencephalomalacia in sheep: some biochemical changes. Can. J. Vet. Res. 53:462-467.

Gould D.H. 2000. Update on sulphur-related polioencephalomalacia. Vet. Clin. North Am., Food Anim. Pract. 16(3):481-496.

Gould D.H., Cummings B.A. \& Hamar D.W. 1997. In vivo indicators of pathologic ruminal sulfide production in steers with diet-induced polioencephalomalacia. J. Vet. Diagn. Invest. 9:72-76.

Gould D.H. 1998. Polioencephalomalacia. J. Anim. Sci. 76:309-314.

Gunn M.F., Baird J.D. \& Wilkie J.S.N. 1987. Accidental sulfur poisoning in a group of Holstein heifers. Can. Vet. J. 28(4):188-192.

Humphries W.R., Phillippo M., Young B.W. \& Bremner I. 1983. The influence of dietary iron and molybdenum on copper metabolism in calves. Brit. J. Nutr. 49:77-86.

Jeffrey M., Duff J.P., Higgins R.J., Simpson V.R., Jackman R., Jones T.O., Mechie S.C. \& Livesey C.T. 1994. Polioencephalomalacia associated with 
the ingestion of ammonium sulphate by sheep and cattle. Vet. Rec. 134(14):343-348.

Jensen R., Griner L.A. \& Adams O.R. 1956. Polioencephalomalacia of cattle and sheep. J. Am. Vet. Med. Assoc. 129:311-321.

Kandylis K. 1984. Toxicology of sulfur in ruminants: review. J. Dairy Sci. 67:2179-2187.

Krasincka B., Gralak M.A., Sieranska B. \& Kulasek G. 1999. The influence of dietary sulphur loading on metabolism and health in young sheep fed low fibre and high starch diet. Reprod Nutr. Dev. 39:625-636.

Kung Jr L. 2008. Burping can be dangerous if you are a ruminant: issues with high sulfur diets. 4th State Dairy Conference, Dubuque, IA.

Lima E.F., Riet-Correa F., Tabosa I.M., Dantas A.F.M. \& Medeiros J.M. 2005. Polioencefalomalacia em caprinos e ovinos na região semi-árida do Nordeste do Brasil. Pesq. Vet. Bras. 25(1):9-14.

Loneragan G.H., Gould D.H., Callan R.J., Sigurdson C.J. \& Hamar D.W. 1998. Association of excess sulfur intake and an increase in hydrogen sulfide concentrations in the ruminal gas cap of recently weaned beef calves with polioencephalomalacia. J. Am. Vet. Med. Assoc. 213(11):1599-1604.

Low J.C., Scott P.R., Howie F., Lewis M., Fitzsimons J. \& Spence J.A. 1996. Sulphur-induced polioencephalomalacia in lambs. Vet. Rec. 138:327-329.

Martin W.B. 1996. Respiratory infections of sheep. Comp. Immunol. Microbiol. Infect. Dis. 19(3):171-179.

McAllister M.M., Gould D.H. \& Hamar D.W. 1992. Sulphide-induced polioencephalomalacia in lambs. J. Comp. Pathol. 106:267-278.

Mayhew I.G. 1989. Large Animal Neurology: a handbook for veterinarians clinicians. Lea and Febiger, Philadelphia. 380p.

Neville B.W., Schauer C.S., Karges K., Gibson M.L., Thompson M.M., Kirschten L.A., Dyer N.W., Berg P.T. \& Lardy G.P. 2010. Effect of thiamine concentration on animal health, feedlot performace, carcass characteristics, and ruminal hydrogen sulfide concentrations in lambs fed diets based on $60 \%$ distillers dried grains plus solubles. J. Anim. Sci. 88:2444-2455.

NRC 2005. Mineral Tolerance of Animals. $2^{\text {nd }}$ ed. National Research Council, National Academy of Sciences, Washington, DC. 385p.

NRC 2007. Nutrient Requeriments of Small Ruminants. National Research Council, National Academy Press, Washington, DC. 362p.

Olkowski A.A., Gooneratne S.R., Rousseaux C.G. \& Christesen D.A. 1992. Role of thiamine status in sulphur induced polioencephalomalacia in sheep. Res. Vet. Sci. 52:78-85.

Pugh D.G. 2004. Clínica de Ovinos e Caprinos. Roca, São Paulo. 468p.

Radostits O.M., Gay C.C., Hinchcliff K.W. \& Constable P.D. 2007. Veterinary Medicine: a textbook of the disease of cattle, sheep, goats, pigs and horses. $10^{\text {th }}$ ed. Saunders Elsevier, Edinburgh. 2065p.

Raisbeck M.F. 1982. Is polioencephalomalacia associated with high-sulfate diets? J. Am. Vet. Med. Assoc. 180(11):1303-1305.
Ramos J.R., Pinho M., Simoes D.L., Baptista A.S. \& Souza P.C.T. 1988. Preparo de colon para operações colorretais eletivas pela ingestão de sulfato de sódio. Revta Bras. Coloproct. 8(2):56-58.

Riebold T.W. 2007. Anesthesia, analgesia, and immobilization of selected species and classes of animals, p.731-747. In: Tranquilli W.J., Thurmon J.C. \& Grimm K.A. (Eds), Lumb and Jones Veterinary Anesthesia and Analgesia. $4^{\text {th }}$ ed. Blackwell Publishing, Iowa.

Rousseaux C.G., Olkowski A.A., Chauvet S.R., Gooneratne S.R. \& Christenson D.A. 1991. Ovine polioencephalomalacia associated with dietary sulphur intake. J. Am. Vet. Med. Assoc. 38:229-239.

Sager R.L., Hamar D.W. \& Gould D.H. 1990. Clinical and biochemical alterations in calves with nutritionally induced polioencephalomalacia. Am. J. Vet Res. 51(12):1969-1974.

Salem G.A., El-Neweshy M.S., Oda S.S., Abou-Olla S.H. \& El-Manakhly E.M. 2010. Experimental sulfur toxicosisin sheep II- Pathologic evaluation of neural and extraneural lesions. Alexandria J. Vet. Sci. 31:97-106.

Sampaio I.B.M. 2010. Estatística aplicada à experimentação animal. 3aㅡ ed. Fundação de Ensino e Pesquisa em Medicina Veterinária e Zootecnia, Belo Horizonte. 264p.

Sant'Ana F.J.F., Nogueira A.P.A., Souza R.I., Cardinal S.G., Lemos R.A.A. \& Barros C.S.L. 2009. Polioencefalomalacia experimental induzida por amprólio em ovinos. Pesq. Vet. Bras. 29(9):747-752.

Scarratt W.K., Collins T.J. \& Sponenberg D.P. 1985. Water deprivation sodium chloride intoxication in a group of feeder lambs. J. Am. Vet. Med. Assoc. 186(9):977-978.

Silva A.M., Flores E.F., Weiblen R., Botton S.A., Irigoyen L.F., Roehe P.M., Brum M.C.S. \& Canto M.C. 1998. Infecção aguda e latente em ovinos inoculados com o herpesvírus bovino tipo 5 (BHV-5). Pesq. Vet. Bras. 18:99-106.

Silva R.R., Silva F.F., Prado I.N., Carvalho G.G.P., Oliveira A.P., Chaves M.A., Mendes F.B.L., Souza D.R. \& Pinheiro A.A. 2007. Effect of the powdered and pelleted rations on the ingestive behavior of Holstein calves. Arch. Zootec. 56(217):227-238.

Suttle N.F. 1991. The interactions between copper, molybdenum and sulphur in ruminant nutrition. Annu. Rev. Nutr. 11:121-140.

Traverso S.D., Colodel E.M., Loretti A.P., Seitz A.L., Correa A.M., Krauspenhar C. \& Driemeier D. 2001. Polioencefalomalacia em bovinos leiteiros no Rio Grande do Sul suplementados com enxofre. $10^{\circ}$ Enapave, Pirassununga, p72. (Resumo)

Tsuka T., Taura Y., Okamura S., Tamura H., Okamoto Y., Okamura Y \& Minami S. 2008. Imaging diagnosis: polioencephalomalacia in a calf. Vet. Radiol. Ultrassound 49(2):149-151.

Vásquez E.F.A., Herrera A.D.N. \& Santiago G.S. 2001. Interação cobre, molibdênio e enxofre em ruminantes. Ciência Rural 31(6):1101:1106.

White J.B. 1964. Sulphur poisoning in ewes. Vet. Rec. 76:278. 